\title{
A practical method for preparation of pneumococcal and nontypeable Haemophilus influenzae inocula that preserves viability and immunostimulatory activity
}

\author{
Lea-Ann S Kirkham ${ }^{1,2^{*}}$, Karli J Corscadden², Selma P Wiertsema ${ }^{1}$, Andrew J Currie ${ }^{1,3}$ and Peter C Richmond ${ }^{1,2}$
}

\begin{abstract}
Background: Convenience is a major reason for using killed preparations of bacteria to investigate host-pathogen interactions, however, host responses to such preparations can result in different outcomes when compared to live bacterial stimulation. We investigated whether cryopreservation of Streptococcus pneumoniae and nontypeable Haemophilus influenzae (NTHi) permitted investigation of host responses to infection without the complications of working with freshly prepared live bacteria on the day of experimental challenge.

Findings: S. pneumoniae and NTHi retained $>90 \%$ viability following cryopreservation in fetal calf serum for at least 8 weeks. Host responses to live, cryopreserved (1 week and 4 weeks), heat-killed or ethanol-killed S. pneumoniae and NTHi were assessed by measuring cytokine release from stimulated peripheral blood mononuclear cells (PBMCs). We found that cryopreserved bacteria, in contrast to heat-killed and ethanol-killed preparations, resulted in comparable levels of inflammatory cytokine release from PBMCs when compared with fresh live bacterial cultures.

Conclusion: Cryopreservation of S. pneumoniae and NTHi does not alter the immunostimulatory properties of these species thereby enabling reproducible and biologically relevant analysis of host responses to infection. This method also facilitates the analysis of multiple strains on the same day and allows predetermination of culture purity and challenge dose.
\end{abstract}

Keywords: Ethanol-killed, Heat-killed, Live bacteria, Cryopreservation, S. pneumoniae, NTHi, Immunostimulation, PBMCS

\section{Background}

Many research laboratories, by necessity, use heat-killed, ethanol-killed, UV-irradiated or paraformaldehyde-fixed bacterial preparations to investigate host-bacterial interactions. However, stimulation of host immune cells with such inactivated preparations can result in very different outcomes when compared with live bacterial stimulation [1-3]. We have shown that in comparison with live Staphylococcus epidermidis, heat-killed and ethanol-killed S. epidermidis preparations have a reduced capacity to

\footnotetext{
* Correspondence: lea-ann.kirkham@uwa.edu.au

'School of Paediatrics and Child Health, The University of Western Australia, 35 Stirling Highway, Perth, WA 6009, Australia

${ }^{2}$ Telethon Institute for Child Health Research, Centre for Child Health

Research, 100 Roberts Road, Perth, WA 6008, Australia

Full list of author information is available at the end of the article
}

activate key innate immune pathways, especially those associated with cytosolic/endosomal bacterial recognition [3]. In addition, Mogensen and colleagues have demonstrated that live but not heat-killed preparations of Streptococcus pneumoniae and Neisseria meningitidis stimulated the host inflammatory response through Toll-like receptor 9 [2].

Convenience is a major reason for using killed preparations of bacteria to investigate host-pathogen interactions. Working with live bacteria usually requires growth to mid-log phase on the day of the stimulation experiment to ensure consistent and reproducible host responses. The time required for mid-log growth on the day of experimentation varies for different bacteria and can take up to 8 hours, which restricts the number of

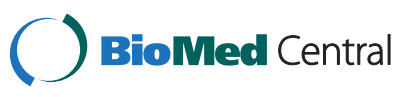


strains that can be assessed on one day and the time available for experimental challenge. In addition, culture contamination is usually only apparent on the day after experimental challenge of the host cells/animal models by checking purity of the culture on an agar plate incubated overnight. An alternative to broth cultures is to harvest bacteria from an overnight agar plate and resuspend in media to the desired optical density, which roughly correlates with bacteria/ml [4]. However, this means that the majority of bacteria are either in stationary phase or indeed dead when used to assess the host response, and results can vary accordingly. We herein describe a simple cryopreservation method using fetal calf serum (FCS) to store mid-log phase S. pneumoniae and NTHi for at least 8 weeks without a significant reduction in viability. We have used a PBMC stimulation assay to demonstrate that preparations of $S$. pneumoniae and NTHi frozen for up to 4 weeks retain the immunostimulatory properties of freshly prepared live bacterial preparations, whereas heat-killed and ethanol-killed preparations do not.

\section{Findings}

There was a dip in viability when $S$. pneumoniae and NTHi were initially frozen, however, both species retained over $90 \%$ viability following 8 weeks cryopreservation (92.6 and $97.0 \%$ respectively when compared with 1 day of cryopreservation), Figure 1 . Viability at 16 weeks cryopreservation was also measured and found to still be $>90 \%$ for both species (data not shown).

With assurance that S. pneumoniae and NTHi remained viable following cryopreservation, we then challenged PBMCs from 5 adult donors with preparations of bacteria that were either frozen for 1 or 4 weeks and compared this with PBMCs challenged with heat-killed,

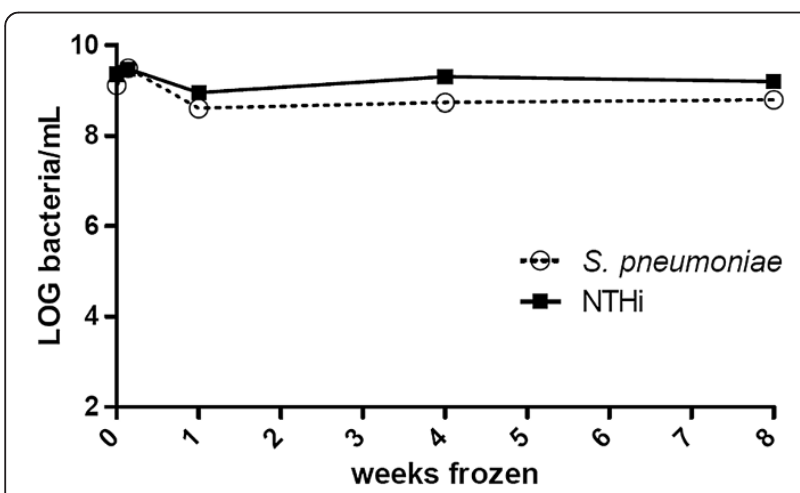

Figure 1 Viability of S. pneumoniae and NTHi over 8 weeks of storage at $-\mathbf{8} 0^{\circ} \mathrm{C}$. The count on day 0 is a total bacteria count using a chamber (bacteria/ml), thereafter viable counts were conducted with the frozen bacteria to give LOG cfu/ml at 1 day, 1 week, 4 weeks and 8 weeks post-freezing. ethanol-killed or live preparations prepared on the day of challenge. PBMC release of 5 inflammatory cytokines was measured as an indication of the immunostimulatory properties of the bacterial preparations. We found that there was no difference in the immunostimulatory properties of frozen S. pneumoniae and NTHi compared with live bacteria regardless of whether the bacteria were stored at $-80^{\circ} \mathrm{C}$ for 1 or 4 weeks (Figure 2). In contrast, stimulation of PBMCs with ethanol-killed preparations resulted in production of significantly lower levels of IL-6, IL-10, TNF $\alpha$ and IL-1 $\beta$ for $S$. pneumoniae, and IFN $\gamma$ and IL- $1 \beta$ for NTHi, when compared with live or frozen preparations (Figure 2, $\mathrm{P}<0.05$ ). Heat-killing retained slightly more immunostimulatory properties than ethanol-killing but there was still reduced immunostimulation in comparison with live or frozen bacteria. No IFNY was released from PBMCs stimulated with either heat or ethanol-killed S. pneumoniae preparations, whereas an average of $200 \mathrm{pg} / \mathrm{ml}$ IFN $\gamma$ was released upon stimulation with live or frozen $S$. pneumoniae. For NTHi, the IL-6, IL-10 and TNF $\alpha$ response was not dependent upon the bacterial treatment with high levels of cytokine production from live, frozen and killed preparations.

\section{Conclusions}

We have described a simple and practical method that enables investigation of live host-pathogen interactions without the restrictions that are normally associated with working with live bacteria such as experimental time, contamination, intra-assay variation and scalability. Serum is a known microbial cryoprotectant [5] and although a similar storage method has been used with S. pneumoniae for challenge of mice [6] we have provided a detailed methodology and clearly demonstrated that cryopreservation of S. pneumoniae and NTHi with FCS preserves the immunostimulatory properties of these species. We have also confirmed that cryopreservation is superior to other methods for standardisation and storage of bacteria that involve inactivation.

Different methods of killing bacteria can alter the immunostimulatory profile of the pathogen either by exposing or destroying PAMPs [1-3]. This was evident in our study where heat and ethanol treatment of S. pneumoniae but not NTHi attenuated the IL- 6 and IL-10 response from PBMCs. This is most likely to be due to the killing treatments destroying key pneumococcal virulence factors such as pneumolysin [1] but not lipooligosaccharide from NTHi, which is heat-stable. This highlights how using killed preparations of bacteria can result in an under or over-stated host immune response to the remaining immunostimulatory components and may result in misleading conclusions about host-pathogen interactions. In summary, we have developed a straightforward and 


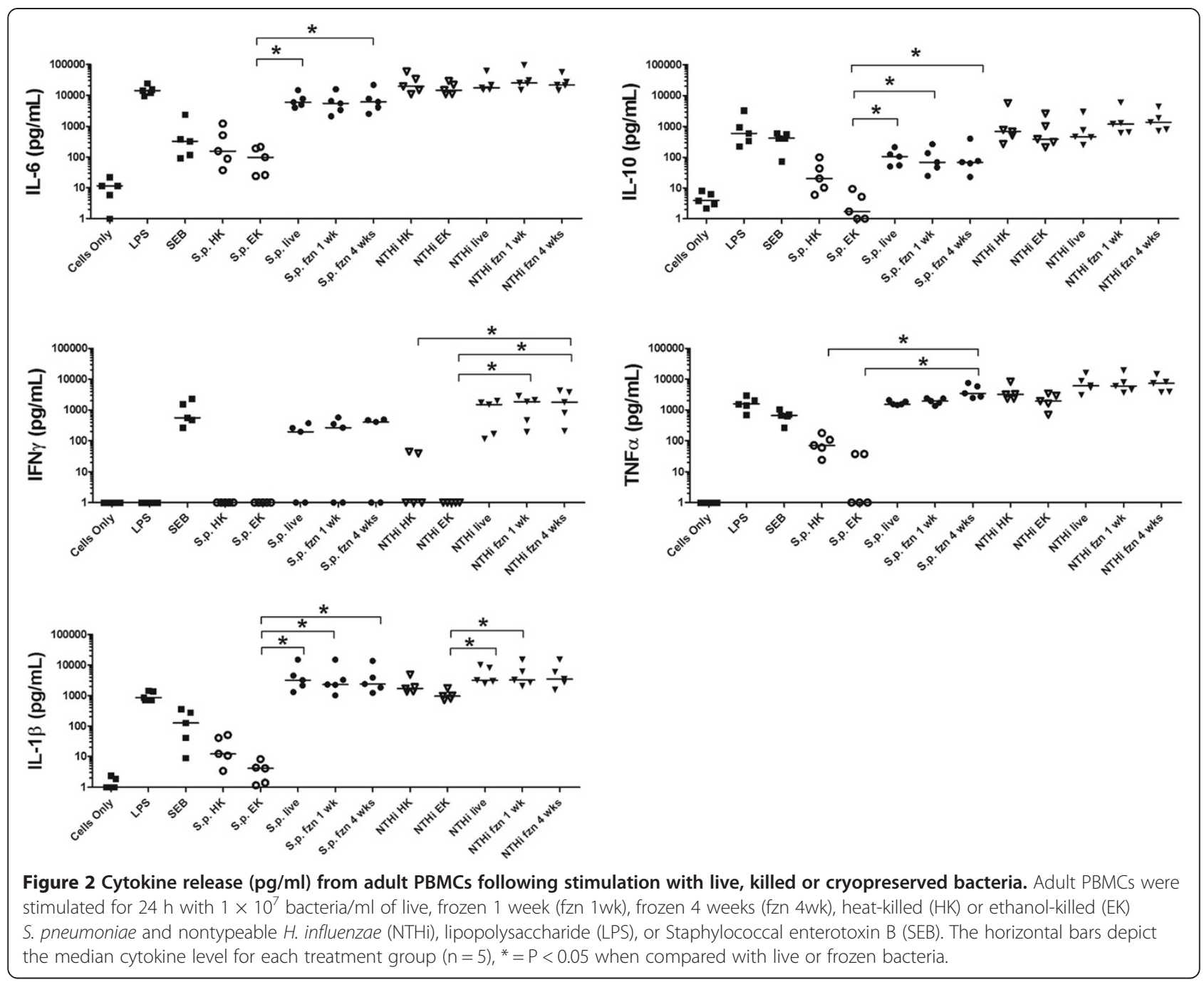

convenient storage method for bacteria and demonstrated that cryopreserved bacteria remain viable for at least 8 weeks and maintain their stimulatory capacities for at least 4 weeks (later time points were not tested). This technique facilitates the analysis of multiple bacterial species on the same day, allows predetermination of culture purity and viability, and most importantly enables accurate investigation of the host response to live bacterial infection.

\section{Methods}

\section{Bacterial strains, culture and cryopreservation}

All reagents were from Sigma Aldrich, New South Wales, Australia unless otherwise stated. Glycerol stocks of S. pneumoniae D39 (ATCC\#7466) and NTHi 289 [7] were streaked out onto blood agar or chocolate agar plates respectively for single colonies and incubated at $37^{\circ} \mathrm{C}$. S. pneumoniae was incubated anaerobically using the $\mathrm{BD}$ GasPak $^{\mathrm{mm}}$ EZ Anaerobic Pouch System [BD Diagnostics,
Australia]. Following overnight incubation, three colonies were selected from each agar plate and used to inoculate culture media. S. pneumoniae was grown statically in brain heart infusion (BHI) broth at $37^{\circ} \mathrm{C}$ and NTHi was grown with shaking in BHI supplemented with $44 \mathrm{~mL} / \mathrm{L}$ glycerol, $30 \mathrm{mg} / \mathrm{L}$ hemin and $10 \mathrm{mg} / \mathrm{L}$ nicotinamide adenine dinucleotide (NAD). Both strains were grown to mid-log phase $\left(\mathrm{OD}_{600 \mathrm{~nm}} 0.55-0.65\right)$, counted at $100 \times$ magnification using a Helber bacteria counting chamber [ProSciTech, Queensland, Australia] and then either subjected to the different treatments (heat, ethanol or freezing) or used fresh on the day of preparation. All cultures were streaked onto agar plates and incubated at $37^{\circ} \mathrm{C}$ overnight to check purity. For cultures that were to be frozen, heat-inactivated FCS [SAFC Biosciences, New South Wales, Australia] was added to the mid-log phase culture to give a final concentration of $20 \% \mathrm{FCS}$ and $1 \mathrm{~mL}$ aliquots were stored in cryovials at $-80^{\circ} \mathrm{C}$. FCS was the only cryoprotectant used for storage of these bacterial stocks. 


\section{Viable counts of frozen bacteria}

Vials of frozen bacterial stocks were thawed at $37^{\circ} \mathrm{C}$ for $2 \mathrm{~min}$ in a water bath, $900 \mu \mathrm{L}$ was transferred to a fresh tube and centrifuged in a benchtop centrifuge at maximum speed for $3 \mathrm{~min}$. The supernatant was discarded and pellet resuspended in $900 \mu \mathrm{L}$ of sterile phosphate buffered saline (PBS) pH 7.4 [Gibco, New South Wales, Australia]. Ten-fold dilutions of each bacteria ranging from $10^{-1}$ to $10^{-6}$ were prepared with PBS in a 96-well polystyrene round bottom plate. Agar plates were divided into 6 sectors, using 2 plates per strain, and three $20 \mu \mathrm{L}$ aliquots of the dilutions were spotted onto each sector. Plates were allowed to dry then incubated overnight at $37^{\circ} \mathrm{C}$. The following day, the number of colony forming units (cfu) per $20 \mu \mathrm{l}$ spot were counted in the sector with approximately $20-80 \mathrm{cfu}$, averaged and multiplied by the dilution factor to give $\mathrm{cfu} / \mathrm{mL}$. Viable counts of cryopreserved bacteria were conducted at 1 day, 1 week, 4 weeks, 8 weeks and 16 weeks postfreezing.

\section{Heat-killing and ethanol-killing of bacteria}

Mid-log phase cultures of S. pneumoniae and NTHi were centrifuged at $3200 \mathrm{~g}$ for $10 \mathrm{~min}$, washed in PBS, and viable counts were conducted as described above. For heat-killing, the bacteria in PBS were incubated in a water bath at $60^{\circ} \mathrm{C}$ for $1 \mathrm{~h}$. For ethanol-killing, the bacteria were resuspended in $70 \%$ ethanol and incubated at $4^{\circ} \mathrm{C}$ for $1 \mathrm{~h}$ with rotation. After killing, bacteria were washed in PBS, aliquoted and stored at $4^{\circ} \mathrm{C}$. Chamber counts were conducted on the killed preparations to determine bacteria/mL and viable counts were conducted to confirm successful killing.

\section{PBMC collection, processing and stimulation with live, frozen, heat-killed and ethanol-killed preparations of bacteria}

Whole blood was collected from five healthy adult donors by peripheral venepuncture and mixed 1:1 with heparinised (2\%) RPMI 1640 media supplemented with 1\% sodium pyruvate, 1\% glutamax, $10 \mathrm{mM}$ HEPES buffer [All Gibco]. The PBMCs were processed and stored as previously described [3]. On the day of stimulation, PBMCs were thawed at $37^{\circ} \mathrm{C}$ for $2 \mathrm{~min}$ in a water bath, added to 2\% FCS RPMI 1640 and centrifuged at $500 \mathrm{~g}$ for $10 \mathrm{~min}$ at room temperature. The supernatant was discarded and cells resuspended in media and counted. The PBMCs were seeded in triplicate for each stimuli at $2.5 \times 10^{5}$ cells per well in 96-well polypropylene roundbottom plates and incubated for $24 \mathrm{~h}$ at $37^{\circ} \mathrm{C}$ in a humidified 5\% $\mathrm{CO}_{2}$ environment. PBMCs were stimulated with either live, heat-killed, ethanol-killed, frozen for 1 week or frozen for 4 week preparations of S. pneumoniae D39 and NTHi 289 at a multiplicity of infection of 10:1 bacteria to cells. Live and frozen bacteria viable counts were determined as described above and total bacterial chamber counts were conducted for the heat- and ethanol-killed preparations. Cells in control wells were stimulated with either PBS (cells only), $1 \mathrm{ng} / \mathrm{mL}$ lipopolysaccharide (LPS, from E. coli R515) [Alexis Biochemicals, Sapphire Biosciences, NSW, Australia] or $1 \mu \mathrm{g} / \mathrm{mL}$ staphylococcal enterotoxin B (SEB, from $S$. aureus). At $24 \mathrm{~h}$ poststimulation, plates were centrifuged for $5 \mathrm{~min}$ at $200 \mathrm{~g}$ and supernatants were harvested, triplicate wells combined and then stored in aliquots at $-80^{\circ} \mathrm{C}$ for subsequent measurement of inflammatory mediators.

\section{Measurement of inflammatory mediators}

IL-6, IL-10, IFN $\gamma$ and TNF $\alpha$ levels $(\mathrm{pg} / \mathrm{mL})$ were measured in cell culture supernatants using a previously described multiplex cytokine bead assay [8]. IL-1 $\beta$ levels in cell supernatants were measured using an ELISA kit [Bender MedSystems, California, USA] and following the manufacturer's instructions, except wells were coated with an alkaline coating buffer $\left(40 \mathrm{mM} \mathrm{NaCo}_{3} ; 70 \mathrm{mM}\right.$ $\mathrm{NaHCO}_{3}$ ) instead of PBS.

\section{Statistical analyses}

PBMC cytokine release following different treatments was compared using a Kruskal-Wallis test with Dunn's post-test analysis using GraphPad Prism [GraphPad Software Inc, California, USA], where $\mathrm{P}<0.05$ was considered statistically significant. For IL- $1 \beta$ one outlier was excluded from analysis due to an out of range maximum value.

\section{Abbreviations \\ Cfu: Colony forming units; EK: Ethanol-killed; HK: Heat-killed; IFN: Interferon; IL: Interleukin; NTHi: Nontypeable Haemophilus influenzae; \\ LPS: Lipopolysaccharide; PAMPs: Pathogen-associated molecular patterns; PBMC: Peripheral blood mononuclear cells; SEB: Staphylococcal enterotoxin B; TNF: Tumour necrosis factor.}

\section{Competing interests}

The authors declare that they have no competing interests.

\section{Authors' contributions}

LK designed the study. LK and SW coordinated the study. KC conducted the viability checks, PBMC stimulations and cytokine measurements. SW, LK, AC and PR contributed to data interpretation and helped draft the manuscript. All authors read and approved the final manuscript.

\section{Acknowledgements}

We would like to thank the laboratory staff at the Vaccine Trials Group for access to the PBMC biobank. NTHi 289 was kindly provided by Professor Jennelle Kyd and S. pneumoniae D39 by Professor James Paton. Funding support for this project was from the University of Western Australia 'near-miss' safety net grant, a Telethon Trust Research Grant and a National Health and Medical Research Council Project Grant \#1011306. Funding bodies did not play a role in any aspects of study design or interpretation.

\section{Author details}

'School of Paediatrics and Child Health, The University of Western Australia, 35 Stirling Highway, Perth, WA 6009, Australia. ${ }^{2}$ Telethon Institute for Child Health Research, Centre for Child Health Research, 100 Roberts Road, Perth, 
WA 6008, Australia. ${ }^{3}$ School of Veterinary and Life Sciences, Murdoch University, South Street, Perth, WA 6150, Australia.

Received: 7 August 2013 Accepted: 4 December 2013 Published: 9 December 2013

\section{References}

1. Malley R, Henneke P, Morse SC, Cieslewicz MJ, Lipsitch M, Thompson CM, Kurt-Jones E, Paton JC, Wessels MR, Golenbock DT: Recognition of pneumolysin by Toll-like receptor 4 confers resistance to pneumococcal infection. Proc Natl Acad Sci USA 2003, 100(4):1966-71.

2. Mogensen TH, Paludan SR, Kilian M, Ostergaard L: Live Streptococcus pneumoniae, Haemophilus influenzae, and Neisseria meningitidis activate the inflammatory response through Toll-like receptors 2, 4, and 9 in species-specific patterns. J Leukoc Biol 2006, 80(2):267-77.

3. Strunk T, Richmond P, Prosser A, Simmer K, Levy O, Burgner D, Currie A: Method of bacterial killing differentially affects the human innate immune response to Staphylococcus epidermidis. Innate Immun 2011, 17(6):508-16.

4. Hessle C, Andersson B, Wold AE: Gram-positive bacteria are potent inducers of monocytic interleukin-12 (IL-12) while gram-negative bacteria preferentially stimulate IL-10 production. Infect Immun 2000, 68(6):3581-6.

5. Hubalek Z: Protectants used in the cryopreservation of microorganisms. Cryobiology 2003, 46(3):205-29.

6. Alexander JE, Lock RA, Peeters CC, Poolman JT, Andrew PW, Mitchell TJ Hansman D, Paton JC: Immunization of mice with pneumolysin toxoid confers a significant degree of protection against at least nine serotypes of Streptococcus pneumoniae. Infect Immun 1994, 62(12):5683-8.

7. Kyd JM, Cripps AW: Potential of a novel protein, OMP26, from nontypeable Haemophilus influenzae to enhance pulmonary clearance in a rat model. Infect Immun 1998, 66(5):2272-2278.

8. de Jager W, te Velthuis H, Prakken BJ, Kuis W, Rijkers GT: Simultaneous detection of 15 human cytokines in a single sample of stimulated peripheral blood mononuclear cells. Clin Diagn Lab Immunol 2003, 10(1):133-9.

doi:10.1186/1756-0500-6-522

Cite this article as: Kirkham et al:: A practical method for preparation of pneumococcal and nontypeable Haemophilus influenzae inocula that preserves viability and immunostimulatory activity. BMC Research Notes 2013 6:522.

\section{Submit your next manuscript to BioMed Central and take full advantage of:}

- Convenient online submission

- Thorough peer review

- No space constraints or color figure charges

- Immediate publication on acceptance

- Inclusion in PubMed, CAS, Scopus and Google Scholar

- Research which is freely available for redistribution 